Post-Print: Published in Journal of Theoretical Biology 2013;331:3847

DOI: http://dx.doi.org/10.1016/j.jtbi.2013.04.010

\title{
Case and partnership reproduction numbers for a curable sexually transmitted infection
}

\author{
Janneke C.M. Heijne ${ }^{\mathrm{a}, *}$, Sereina A. Herzoga ${ }^{\mathrm{a}}$, Christian L. Althaus ${ }^{\mathrm{a}}$, Nicola Low ${ }^{\mathrm{a}}$, Mirjam \\ Kretzschmar $^{\mathrm{b}, \mathrm{c}}$ \\ ${ }^{a}$ Institute of Social and Preventive Medicine (ISPM), University of Bern, Finkenhubelweg 11, 3012 Bern, Switzerland \\ ${ }^{b}$ Julius Centre for Health Sciences and Primary Care, University Medical Centre Utrecht, Heidelberglaan 100, 3584 \\ CX, Utrecht, the Netherlands \\ ${ }^{c}$ Centre for Infectious Disease Control, Epidemiology and Surveillance Unit, RIVM, PO Box 1, 3720 BA Bilthoven, \\ the Netherlands
}

\begin{abstract}
Sexually transmitted infections (STIs) are, by definition, transmitted between sexual partners. For curable STIs an infected index case can potentially re-infect the same partner multiple times. Thus, $R_{0}$, the average number of secondary infections one typical infected individual will produce during his or her infectious period is not necessarily the same as the average number of secondary cases (infected persons). Here we introduce the new concept of the case reproduction number $\left(R_{c}\right)$. In addition, we define the partnership reproduction number $\left(R_{p}\right)$ as the average number of secondary partnerships consisting of two infected individuals one typical infected individual will produce over his or her infectious lifetime. $R_{p}$ takes into account clearance and re-infection within partnerships, which results in a prolongation of the duration of the infectious period. The two new reproduction numbers were derived for a deterministic pair model with serial monogamous partnerships using infection parameters for Chlamydia trachomatis, an example of a curable STI. We showed that re-infection within partnerships means that curable STIs can be sustained endemically even when the average number of secondary cases a person produces during his or her infectious period is below one.
\end{abstract}

Keywords: Basic reproduction number, Disease Transmission: Infectious, Deterministic model, Sexually Transmitted Infections, Chlamydia trachomatis.

\footnotetext{
${ }^{*}$ Corresponding author at: Institute of Social and Preventive Medicine (ISPM), University of Bern, Finkenhubelweg 11, 3012 Bern, Switzerland. Tel: +31 64976 0512. Fax: +41 316313520

Email address: jheijne@ ispm.unibe.ch (Janneke Heijne)
} 


\section{Introduction}

The basic reproduction number $\left(R_{0}\right)$ is widely used in infectious disease epidemiology and mathematical modeling to determine whether an infectious disease can invade a susceptible population (Anderson and May, 1982; Diekmann and Heesterbeek, 2000; Ferguson et al., 2003). $R_{0}$ is defined as the average number of secondary infections one typical infected individual will produce during his or her infectious period in a totally susceptible population (Anderson and May, 1991; Dietz, 1975). This number has important epidemiological threshold properties but does not distinguish between the number of secondary infection events and the number of secondarily infected individuals (cases).

Mathematical models that have been used to derive $R_{0}$ often assume that contacts between individuals are instantaneous with no repeated contacts between the same individuals. However, sexually transmitted infections (STIs) are predominantly transmitted within a partnership of two individuals who engage in sexual intercourse and have repeated sexual contacts with each other. For curable STIs (Susceptible-Infected(-Recovered)-Susceptible type), an index case and their partner can potentially re-infect each other more than once within a partnership (Fig. 1). Therefore, the average number of secondary infections $\left(R_{0}\right)$ does not necessarily equal the average number of secondary cases an infected individual will produce during his or her infectious period.

Several models have been used to derive $R_{0}$ for curable STIs and allow for re-infection (Kretzschmar et al., 1994; Chick et al., 2000; Lloyd-Smith et al., 2004). These studies did not, however, distinguish between the number of secondary infections and the number of secondary cases. Other studies have derived $R_{0}$ using models that take into account partnership duration explicitly (Diekmann et al., 1991; Kretzschmar and Dietz, 1998; Diekmann et al., 1998; Britton et al., 2007) but these are for Susceptible-Infected (SI) infections (for example HIV) where an index case can infect the susceptible partner only once.

In order to account for an infection in which an index case can infect a partner more than once we define the average number of secondary cases a primary case will infect during his or her infectious period as the case reproduction number $R_{c}$ (Table 1). We also define the average number 
of secondary partnerships consisting of two infected individuals one typical infected individual will produce during his or her infectious lifetime. We call this the partnership reproduction number $\left(R_{p}\right)$ (Table 1)

Chlamydia trachomatis (chlamydia) is an example of an endemic curable STI, which is the most common notifiable infection in some developed countries (Centers for Disease Control and Prevention (CDC), 2010). It has a long infectious period (Althaus et al., 2010) and most infections are asymptomatic in both men and women. Untreated chlamydia can ascend to the upper genital tract and, in women, cause pelvic inflammatory disease (PID), which can lead to ectopic pregnancy and infertility (Holmes et al., 2008). People testing positive and treated for chlamydia are at high risk of repeat infection (Batteiger et al., 2010) through re-infection within the existing partnership if the partner was not adequately treated, infection from a new partner, or from treatment failure.

Earlier attempts to estimate $R_{0}$ for chlamydia have shown varying results (Brunham et al., 1994; Jolly and Wylie, 2002; Stigum et al., 1994). Most estimates have been based on the formula $R_{0}=\beta c D$, where $\beta$ is the transmission probability per partnership, $c$ the partner change rate and $D$ the duration of the infectious period. Brunham et al. (1994) obtained values for $R_{0}$ greater than 1 (1.43 and 3.6), based on a sexual behaviour study from Nairobi, Kenya. Jolly and Wylie (2002) used sex partner data from the Canadian notifiable STI registry and found values for $R_{0}$ below 1 for individuals with lower partner change rates (0.7) and above 1 for those with higher rates (1.09). Stigum et al. (1994) used an instantaneous contact model and estimated $R_{0}>1$ when assuming mixing between core and non-core groups, based on Norwegian sexual survey data.

In the field of STI prevention, contact tracing is an important intervention to control the spread of curable STIs. Empirical data about the infection status of actively sought sexual partners of a diagnosed infected individual are recorded and can be used to calculate a reproduction number. Using the definitions above, only the case reproduction number $\left(R_{c}\right)$ can be calculated from contact tracing data because the direction of transmission from infected partners is usually unknown and traced partners might already have been infected more than once by the same partner and could have cleared the infection naturally before diagnosis. In one study that used contact tracing data to calculate reproduction numbers for chlamydia Potterat et al. (1999), the authors found an overall 
reproduction number of 0.55 and numbers between 0.19 and 2.29 for different subgroups. They report these as basic reproduction numbers $\left(R_{0}\right)$ and interpret the overall $R_{0}<1$ as evidence for the success of chlamydia control measures in their study region.

In this paper we derive expressions for the case and partnership reproduction number $\left(R_{c}\right.$ and $R_{p}$ ) for a curable STI using a pair model with serial monogamous partnerships. We then show how $R_{c}$ and $R_{p}$ are related to the basic reproduction number $R_{0}$ and derive the epidemic threshold values of all three reproduction numbers. Finally, we use the different reproduction numbers to investigate the contribution of re-infection within partnerships to the transmission and endemicity of chlamydia.

\section{The model}

Pair models for transmission of STIs were first described by Dietz and Hadeler (1988) and adopted by many others (Kretzschmar et al., 1994; Kretzschmar and Dietz, 1998; Xiridou et al., 2003; Van de Velde et al., 2010; Chen and Ghani, 2010; Heijne et al., 2011). The model used here has been described in detail elsewhere (Heijne et al., 2011). In brief, the model describes a population where people are single $(X)$ or in a partnership $(P)$. Individuals can form a partnership at any time (with rate $\rho$ ), and break up at a rate $\sigma$ (Table 2). Partnerships also break up when one of the partners dies; the duration of the sexually active life is $1 / \mu$. From here onwards we use the term separation, which includes breaking up for either reason. Pair formation and separation can be described by the following set of ordinary differential equations

$$
\begin{aligned}
& \frac{d X}{d t}=\mu+2(\sigma+\mu) P-(\rho+\mu) X \\
& \frac{d P}{d t}=\frac{1}{2} \rho X-(\sigma+2 \mu) P
\end{aligned}
$$

At steady state, the proportion of singles $(X)$ equals $\frac{2 \mu+\sigma}{2 \mu+\rho+\sigma}$, and the proportion of people in a partnership $(2 P)$ is $\frac{\rho}{2 \mu+\rho+\sigma}$. This relationship can be used to estimate the pair formation rate and separation rate from empirical data. Here, the sexual behavioural parameters are informed by data from a nationally representative sample of the UK general population (Natsal-2) (Johnson et al., 2001). The behavioural parameters are taken from participants aged $16-24$ years, so the duration 
of sexually active life $(1 / \mu)$ is set to 9 years. We assume an average of 1.5 new partners per year, and that $70 \%$ of the population is in a partnership at any time. By solving $2 P=\frac{\rho}{2 \mu+\rho+\sigma}=0.7$ and $\rho X=\rho \frac{2 \mu+\sigma}{2 \mu+\rho+\sigma}=1.5$ we obtain a pair formation rate of 5 per year and a pair separation rate 1.9 per year (Table 2), resulting in an average partnership duration of 0.5 year and average gap duration of 0.2 year. We assumed the partnership and gap duration to be exponentially distributed taking into account that some partnerships and gaps are very short, whereas others can be long.

The infection states of the model are susceptible (S), asymptomatically infected (I) and recovered (R). Transmission only happens within pairs, where $\beta$ is the transmission probability per sex act, and $\phi$ the frequency of sex acts. Individuals can clear the infection naturally, with $1 / \gamma_{f}$ being the duration of infection in women, and $1 / \gamma_{m}$ the duration of infection in men. After a period of immunity $(1 / \epsilon)$ individuals can become susceptible again. The transmission process and the pair process are independent and can be described by a set of ordinary differential equations. We use $f$ for women and $m$ for men. For example, $X_{f, S}$ is a single susceptible woman, and $X_{m, S}$ is a single susceptible man. In the formulation of pairs, the first subscript denotes the infection status of the woman, and the second one the infection status of the man. The derivations of the reproduction numbers use different values for the infectious duration in women and men because men are more likely to be treated for symptomatic infection than women (Holmes et al., 2008). The derivations are described from the point of view of a woman, because intervention measures for controlling the transmission of STIs are often targeted towards women only (Centers for Disease Control and Prevention, 2010). 


$$
\begin{aligned}
\frac{d X_{f, S}}{d t} & =0.5 \mu+(\sigma+\mu)\left(P_{S S}+P_{S I}+P_{S R}\right)-(\rho+\mu) X_{f, S}+\epsilon X_{f, R} \\
\frac{d X_{f, I}}{d t} & =(\sigma+\mu)\left(P_{I S}+P_{I I}+P_{I R}\right)-\left(\rho+\mu+\gamma_{f}\right) X_{f, I} \\
\frac{d X_{f, R}}{d t} & =(\sigma+\mu)\left(P_{R S}+P_{R I}+P_{R R}\right)-(\rho+\mu+\epsilon) X_{f, R}+\gamma_{f} X_{f, I} \\
\frac{d X_{m, S}}{d t} & =0.5 \mu+(\sigma+\mu)\left(P_{S S}+P_{I S}+P_{R S}\right)-(\rho+\mu) X_{m, S}+\epsilon X_{m, R} \\
\frac{d X_{m, I}}{d t} & =(\sigma+\mu)\left(P_{S I}+P_{I I}+P_{R I}\right)-\left(\rho+\mu+\gamma_{m}\right) X_{m, I} \\
\frac{d X_{m, R}}{d t} & =(\sigma+\mu)\left(P_{S R}+P_{I R}+P_{R R}\right)-(\rho+\mu+\epsilon) X_{m, R}+\gamma_{m} X_{m, I} \\
\frac{d P_{S S}}{d t} & =2 \rho \frac{X_{f, S} X_{m, S}}{X}-(\sigma+2 \mu) P_{S S}+\epsilon\left(P_{S R}+P_{R S}\right) \\
\frac{d P_{S I}}{d t} & =2 \rho \frac{X_{f, S} X_{m, I}}{X}-\left(\sigma+2 \mu+\beta \phi+\gamma_{m}\right) P_{S I}+\epsilon P_{R I} \\
\frac{d P_{S R}}{d t} & =2 \rho \frac{X_{f, S} X_{m, R}}{X}-(\sigma+2 \mu+\epsilon) P_{S R}+\gamma_{m} P_{S I}+\epsilon P_{R R} \\
\frac{d P_{I S}}{d t} & =2 \rho \frac{X_{f, I} X_{m, S}}{X}-\left(\sigma+2 \mu+\beta \phi+\gamma_{f}\right) P_{I S}+\epsilon P_{I R} \\
\frac{d P_{I I}}{d t} & =2 \rho \frac{X_{f, I} X_{m, I}}{X}-\left(\sigma+2 \mu+\gamma_{f}+\gamma_{m}\right) P_{I I}+\beta \phi P_{S I}+\beta \phi P_{I S} \\
\frac{d P_{I R}}{d t} & =2 \rho \frac{X_{f, I} X_{m, R}}{X}-\left(\sigma+2 \mu+\gamma_{f}+\epsilon\right) P_{I R}+\gamma_{m} P_{I I} \\
\frac{d P_{R S}}{d t} & =2 \rho \frac{X_{f, R} X_{m, S}}{X}-(\sigma+2 \mu+\epsilon) P_{R S}+\gamma_{f} P_{I S}+\epsilon P_{R R} \\
\frac{d P_{R I}}{d t} & =2 \rho \frac{X_{f, R} X_{m, I}}{X}-\left(\sigma+2 \mu+\gamma_{m}+\epsilon\right) P_{R I}+\gamma_{f} P_{I I} \\
\frac{d P_{R R}}{d t} & =2 \rho \frac{X_{f, R} X_{m, R}}{X}-(\sigma+2 \mu+2 \epsilon) P_{R R}+\gamma_{f} P_{I R}+\gamma_{m} P_{R I} \\
& =
\end{aligned}
$$

where

$$
\begin{aligned}
P & =P_{S S}+P_{S I}+P_{S R}+P_{I S}+P_{I I}+P_{I R}+P_{R S}+P_{R I}+P_{R R} \\
X_{f} & =X_{f, S}+X_{f, I}+X_{f, R} \\
X_{m} & =X_{m, S}+X_{m, I}+X_{m, R} \\
N & =X_{f}+X_{m}+2 P=1
\end{aligned}
$$


We assumed a frequency of unprotected sex acts of once a week. By setting the derivatives to zero and assuming equal natural clearance rate between men and women, we obtained a solution for $\beta$ as a function of all other parameters including the prevalence (AppendixA). The prevalence is defined as: $\left(X_{f, I}+X_{m, I}+P_{S I}+P_{I S}+P_{I R}+P_{R I}+2 P_{I I}\right) / N$.

\section{The reproduction numbers}

\subsection{The case reproduction number $\left(R_{c}\right)$}

The case reproduction number $\left(R_{c}\right)$ is defined as the average number of secondary cases one typical infected individual will produce during his or her infectious period starting in a partnership with two infected individuals in a totally susceptible population (Table 1). Here, we use the term cases in a broad sense, indicating all infected individuals and not only those individuals with a diagnosed or laboratory confirmed infection.

We start by deriving an expression for the female case reproduction number $\left(R f_{c}\right)$ considering the entire course of infection of an infected woman (see white boxes in Fig. 2 for a graphical illustration of the infectious states of a woman). We assume that a woman starts her infectious life as part of a pair with an infected man, that is, in state $P_{I I}$. To compute $R f_{c}$, we need the probability that the woman is still infected when separating from a partner, the probability that she is still infected when she forms a new partnership, the probability that she transmits to her new partner, and the number of new partners during her remaining infectious period (Fig. 1).

We first derive an expression for the probability that the woman is still infected when separating from a $P_{I I}$ partnership. A woman can reach the state $X_{f, I}$ either directly by separation from the $P_{I I}$ partnership (Eq. 4) or indirectly, by first passing through $P_{I R}$ or $P_{I S}$ partnership status before separation (Eq. 5), or by going through $i$ loops of clearance of the male partner and re-infection of the male partner by the female (Eq. 9). The probability of immediate separation is given by

$$
\mathbf{P}\left(P_{I I} \rightarrow X_{f, I}\right)=\frac{\sigma+\mu}{\sigma+2 \mu+\gamma_{f}+\gamma_{m}}
$$




$$
\begin{aligned}
\sum_{i=1}^{\infty} r_{f}^{i} & =\frac{r_{f}}{1-r_{f}} \\
& =\frac{\beta \phi \gamma_{m} \epsilon}{\left(\sigma+2 \mu+\gamma_{f}\right)\left(\left(\sigma+2 \mu+\gamma_{f}+\gamma_{m}\right)\left(\sigma+2 \mu+\gamma_{f}+\epsilon\right)+\beta \phi\left(\sigma+2 \mu+\gamma_{f}+\gamma_{m}+\epsilon\right)\right)}
\end{aligned}
$$

$$
\begin{aligned}
& =\left(\frac{\gamma_{m}}{\sigma+2 \mu+\gamma_{f}+\gamma_{m}}\right)\left(\frac{\sigma+\mu}{\sigma+2 \mu+\gamma_{f}+\epsilon}\right) \\
& +\left(\frac{\gamma_{m}}{\sigma+2 \mu+\gamma_{f}+\gamma_{m}}\right)\left(\frac{\epsilon}{\sigma+2 \mu+\gamma_{f}+\epsilon}\right)\left(\frac{\sigma+\mu}{\sigma+2 \mu+\gamma_{f}+\beta \phi}\right)
\end{aligned}
$$

We define $q_{0}$ as the probability of still being infected after separation when there is no re-infection within the partnership. This is the sum of Eq. 4 and Eq. 5

$$
q_{0 f}=\frac{\sigma+\mu}{\sigma+2 \mu+\gamma_{f}+\gamma_{m}}\left(1+\frac{\gamma_{m}}{\sigma+2 \mu+\gamma_{f}+\epsilon}\left(1+\frac{\epsilon}{\sigma+2 \mu+\gamma_{f}+\beta \phi}\right)\right)
$$

Now, we consider the situation that one or more re-infections of the male partner take place before the pair separates. Let $b_{f}$ be the probability that the woman transmits the infection to her susceptible partner

$$
b_{f}=\mathbf{P}\left(P_{I S} \rightarrow P_{I I}\right)=\frac{\beta \phi}{\sigma+2 \mu+\gamma_{f}+\beta \phi}
$$

The probability that the male partner of a $P_{I I}$ pair clears his infection and is then re-infected by the female index case is denoted by $r_{f}$

$$
\begin{aligned}
r_{f} & =\mathbf{P}\left(P_{I I} \rightarrow P_{I R}\right) \mathbf{P}\left(P_{I R} \rightarrow P_{I S}\right) \mathbf{P}\left(P_{I S} \rightarrow P_{I I}\right) \\
& =\left(\frac{\gamma_{m}}{\sigma+2 \mu+\gamma_{f}+\gamma_{m}}\right)\left(\frac{\epsilon}{\sigma+2 \mu+\gamma_{f}+\epsilon}\right)\left(\frac{\beta \phi}{\sigma+2 \mu+\gamma_{f}+\beta \phi}\right)
\end{aligned}
$$

Then $r_{f}{ }^{i}$ is the probability that clearance and re-infection of the male partner happens exactly $i$ times before separation of the pair, $i \in \mathbb{N}$. The probability that at least one re-infection occurs is given by 
Now the probability that a woman who started in a $P_{I I}$ partnership is still infected after separation from that partner is given by

$$
q_{0 f}\left(1+\frac{r_{f}}{1-r_{f}}\right)=\frac{q_{0 f}}{1-r_{f}}=\frac{\mu+\sigma}{\sigma+2 \mu+\gamma_{f}}
$$

Because all parameters are positive, this quantity is $<1$.

While the woman is single she leaves the infected state with rate $\gamma_{f}+\mu$ before forming a new partnership. The probability that she is still infectious and sexually active when she meets her next partner is

$$
d_{f}=\frac{\rho}{\rho+\gamma_{f}+\mu}
$$

For each partner we have to compute the probability that the woman is still infectious and sexually active when forming the partnership assuming that every new partnership is with a susceptible. For the $i$-th partner this is $\left(d_{f} \frac{q_{0 f}}{1-r_{f}}\right)^{i}$. Therefore, she will infect

$$
\begin{aligned}
R f_{c} & =b_{f} \sum_{i=1}^{\infty}\left(d_{f} \frac{q_{0 f}}{1-r_{f}}\right)^{i}=\frac{b_{f} q_{0 f} d_{f}}{1-q_{0 f} d_{f}-r_{f}} \\
& =\frac{\beta \phi \rho(\sigma+\mu)}{\left(\gamma_{f}+\mu\right)\left(\sigma+2 \mu+\gamma_{f}+\beta \phi\right)\left(\sigma+2 \mu+\gamma_{f}+\rho\right)}
\end{aligned}
$$

new partners during her infectious period.

The derivation of the case reproduction number $R m_{c}$ of males towards the female population is analogous. The case reproduction number for the entire transmission cycle is then given by the geometric mean of $R f_{c}$ and $R m_{c}$ (Diekmann and Heesterbeek, 2000)

$$
R_{c}=\sqrt{R f_{c} R m_{c}}
$$

\subsection{The partnership reproduction number $\left(R_{p}\right)$}

The partnership reproduction number $\left(R_{p}\right)$ builds on the concept of $R_{c} . R_{p}$ is defined as the average number of secondary $P_{I I}$ partnerships one typical infected individual will produce during his or her infectious lifetime starting in a $P_{I I}$ partnership in a totally susceptible population. In con- 
structing this reproduction number, we assume that re-infection of the index case in a partnership prolongs the initial infectious period of the index case; the prolonged infectious period is called the infectious lifetime (Table 1 and Fig. 1). As above, we use the probabilities that a person is still infected when forming a new partnership and when separating from the partnership. Additionally, we include in the probability of still being infected after separation, a term that involves clearance and re-infection of the index case in the partnership.

We follow again an infected woman who starts her infectious life in a partnership with an infected man. We denote by $s_{f}$ the probability that the female index case clears the infection in a partnership and is re-infected by her male partner before separation (dashed arrows in Fig. 1 and 2)

$$
\begin{aligned}
s_{f} & =\mathbf{P}\left(P_{I I} \rightarrow P_{R I}\right) \mathbf{P}\left(P_{R I} \rightarrow P_{S I}\right) \mathbf{P}\left(P_{S I} \rightarrow P_{I I}\right) \\
& =\left(\frac{\gamma_{f}}{\sigma+2 \mu+\gamma_{f}+\gamma_{m}}\right)\left(\frac{\epsilon}{\sigma+2 \mu+\gamma_{m}+\epsilon}\right)\left(\frac{\beta \phi}{\sigma+2 \mu+\gamma_{m}+\beta \phi}\right)
\end{aligned}
$$

Before separation, a $P_{I I}$ pair can go through $i$ cycles of clearance and re-infection, where either the male or female partner can clear the infection and become re-infected. The probability of this happening is given by

$$
\sum_{k=0}^{i}\left(\begin{array}{l}
i \\
k
\end{array}\right) r_{f}^{k} s_{f}^{i-k}
$$

and therefore the probability that at least one re-infection occurs before separation of the pair is given by

$$
\sum_{i=1}^{\infty} \sum_{k=0}^{i}\left(\begin{array}{l}
i \\
k
\end{array}\right) r_{f}{ }^{k} s_{f}^{i-k}=\frac{r_{f}+s_{f}}{1-\left(r_{f}+s_{f}\right)}
$$

Eq. 16 holds because $r_{f}+s_{f}<1$. The probability that a woman who started in a $P_{I I}$ partnership is still infected after separation from that partner is given by

$$
q_{0 f}\left(1+\frac{r_{f}+s_{f}}{1-\left(r_{f}+s_{f}\right)}\right)=\frac{q_{0 f}}{1-\left(r_{f}+s_{f}\right)}
$$

This quantity is also $<1$, analogous to Eq. 10 .

The number of $P_{I I}$ partnerships caused by an infected women in a $P_{I I}$ partnership during her 
entire infectious lifetime $\left(R f_{p}\right)$ is given by

$$
R f_{p}=b_{f} \sum_{i=1}^{\infty}\left(d_{f} \frac{q_{0 f}}{1-\left(r_{f}+s_{f}\right)}\right)^{i}=\frac{b_{f} q_{0 f} d_{f}}{1-q_{0 f} d_{f}-\left(r_{f}+s_{f}\right)}
$$

The sum converges because the expression in the bracket fulfills the condition to be less than one (see Eq. 11 and 17) and $R f_{p} \geq 0$.

Again, the derivation of the number of $P_{I I}$ partnerships produced by an infected man in a $P_{I I}$ partnership during his entire infectious lifetime $\left(R m_{p}\right)$ is analogous and the overall partnership reproduction number $\left(R_{p}\right)$ is again the geometric mean of the two quantities.

\subsection{The basic reproduction number $\left(R_{0}\right)$}

The concept of $R_{0}$ has been formalised by Diekmann and Heesterbeek (2000). Here, we apply this concept to calculate $R f_{0}$ for the pair model, i.e. the average number of secondary infections one typical infected woman will produce during her infectious period starting in a $P_{I I}$ partnership in a totally susceptible population. In the model, there is one infection state, but 4 subpopulations of women who are in the infection state at a given time: single (1); in a pair with a susceptible partner (2); in a pair with an infected partner (3) and in a pair with a recovered partner (4) (Fig. 2). We can now write down the matrix $\mathbf{G}$ where the $i j$-th element describes the transitions from state $j$ to state $i$

$$
\mathbf{G}=\left(\begin{array}{cccc}
-\left(\rho+\mu+\gamma_{f}\right) & \sigma+\mu & \sigma+\mu & \sigma+\mu \\
\rho & -\left(\sigma+2 \mu+\gamma_{f}+\beta \phi\right) & 0 & \epsilon \\
0 & \beta \phi & -\left(\sigma+2 \mu+\gamma_{f}+\gamma_{m}\right) & 0 \\
0 & 0 & \gamma_{m} & -\left(\sigma+2 \mu+\gamma_{f}+\epsilon\right)
\end{array}\right)
$$

Some transitions in the matrix cannot occur. For example, there is no transition from state 1 to states 3 and 4 (i.e. $\mathbf{G}_{31}=\mathbf{G}_{41}=0$ ) because we assume that partnerships do not start with a sexual contact and that every new partnership is with a susceptible individual.

The $i j$-th element of the matrix $-\left(\mathbf{G}^{-1}\right)$ gives the expected time a woman who presently has state $j$ will spend in state $i$ during her infectious period. Similar as with the other reproduction 
numbers, we start with an infected woman in a pair with an infected man (state 3), and we determine the time she spends in state 2 with a susceptible partner during her infectious lifetime (i.e. $\left.\left(-\mathbf{G}^{-1}\right)_{23}\right)$. The total number of infections a woman will produce while she is together with a susceptible man during her infectious period is then given by

$$
\begin{aligned}
R f_{0} & =\beta \phi\left(-\mathbf{G}^{-1}\right)_{23} \\
& =\frac{\beta \phi\left(\rho(\sigma+\mu)(\sigma+2 \mu+\epsilon)+\gamma_{f}\left(\gamma_{m} \epsilon+\rho(\sigma+\mu)\right)+\gamma_{m}(\epsilon(\rho+\mu)+\rho(\sigma+\mu))\right)}{\left(\gamma_{f}+\mu\right)\left(\sigma+2 \mu+\gamma_{f}+\rho\right)\left(\left(\sigma+2 \mu+\gamma_{f}+\gamma_{m}\right)\left(\sigma+2 \mu+\gamma_{f}+\epsilon\right)+\beta \phi\left(\sigma+2 \mu+\gamma_{f}+\gamma_{m}+\epsilon\right)\right)}
\end{aligned}
$$

The calculation of the male basic reproduction number is analogous and the basic reproduction number for the entire transmission cycle $\left(R_{0}\right)$ is also given by the geometric mean of the two. In a SI model (i.e $\gamma_{f}=\gamma_{m}=0$ and $\epsilon=0$ ), the equation for $R_{0}$ is the same as the equation for $R_{c}$ and similar to the equation described by Diekmann et al. (1991) on page 333.

\subsection{Relationship between reproduction numbers}

We can rewrite the expression for $R f_{0}$ using the same probabilities as described in the previous sections. We then obtain the following expression

$$
\begin{aligned}
R f_{0} & =\frac{b_{f} q_{0 f} d_{f}}{1-q_{0 f} d_{f}-r_{f}}+\frac{r_{f}}{1-r_{f}}\left(\frac{b_{f} q_{0 f} d_{f}}{1-q_{0 f} d_{f}-r_{f}}+1\right) \\
& =R f_{c}+\frac{r_{f}}{1-r_{f}}\left(R f_{c}+1\right)
\end{aligned}
$$

where $\frac{r_{f}}{1-r_{f}}\left(R f_{c}+1\right)$ describes the expected number of re-infections in the starting partnership and in the subsequent partnerships. This term, divided by $R f_{0}$, gives the fraction of the female reproduction number that is attributable to re-infection of the partner by the index case. It can be seen that $R f_{0}$ is always $\geq R f_{c}$ and when there is no re-infection within partnerships (i.e. $r_{f}=0$ ), $R f_{0}=R f_{c}$.

The equation for $R f_{p}$ can also be written in terms of $R f_{c}$

$$
R f_{p}=R f_{c}\left(1+\frac{s_{f}}{1-q_{0 f} d_{f}-r_{f}-s_{f}}\right)
$$

where the first term of the equation is the number of secondary cases a women produces in her 
first infectious period, and the second term describes the number of secondary cases in subsequent infectious periods as a result of clearance and re-infection of the female index case in a partnership. Note that $R f_{p}$ is always $\geq R f_{c}$ but not necessarily always $\geq R f_{0}$ and that when the probability of re-infection of the index case is set to zero (i.e. $s_{f}=0$ ), $R f_{p}$ equals $R f_{c}$.

When there is no re-infection possible (for example because of lifelong infection or immunity or because contacts are instantaneous) $r_{f}$ and $s_{f}$ are zero, and all reproduction numbers equal the average number of secondary cases $\left(R f_{c}\right)$. When assuming instantaneous contacts the equations for $R f_{c}$ can be reduced to the formula $\beta c D$, where $\beta$ is the transmission probability per partnership, $c$ the partner change rate and $D$ the duration of the infectious period. Assuming instantaneous contacts result in an infinitely large separation rate $\sigma$, Eq. 12 can then be written as

$$
R f_{0}=R f_{c}=\beta \cdot \rho \cdot \frac{1}{\gamma_{f}+\mu}
$$

where $\rho$ represents the partner change rate per year, $\frac{1}{\gamma_{f}+\mu}$ the average female duration of being infectious in years, $\beta$ the transmission probability per contact assuming that $\frac{\phi}{\sigma}$ (i.e. the number of sex acts per partnership) equals 1 . We have also provided derivations for the reproduction numbers for heterogeneous sexual behaviour (AppendixB).

\subsection{Epidemic threshold values}

By definition, the threshold value above which the infection can be sustained endemically, is given by $R_{0}=1$. Threshold values for the other reproduction numbers can be derived using Eq. 21 and 22. If the contribution of re-infections is large enough to ensure that $R_{0}>1$, Eq. 21 shows that an infection can be present endemically even when $R_{c}<1$. This means that if we can estimate $R_{c}$ from contact tracing data, we still need information about the impact of reinfections on disease dynamics to judge the transmission potential of the infection. Also, knowing that $R_{c}<1$ is not sufficient to conclude that an intervention has been sufficiently effective to eventually stop transmission. The relationship between $R_{0}$ and $R_{c}$ can provide information on the impact that preventing re-infections within partnerships can contribute to intervention success. 
Assuming that $R_{0}=1$ we can derive the following threshold equation for $R_{c}$

$$
\sqrt{\left(\left(1-r_{f}\right) R f_{0}-r_{f}\right)\left(\left(1-r_{m}\right) R m_{0}-r_{m}\right)}
$$

Under the condition that $R f_{0}=R m_{0}=1$, the equation simplifies to $\sqrt{\left(1-2 r_{f}\right)\left(1-2 r_{m}\right)}$. The equation can be simplified further to the intuitive formula of $1-2 r$, when assuming that men and women are equal (i.e. $r_{f}=r_{m}=r$ and $s_{f}=s_{m}=s$ ). When $r$ is set to zero, $R_{c}$ has also a threshold value of 1 . Because $R_{c} \geq 0$ it follows that $r$ is always $\leq 0.5$. Using the epidemic threshold value for $R_{c}$, we obtain for $R_{p}$ the simplified threshold value of $(1-2 r)\left(1+\frac{s}{1-d q_{0}-r-s}\right)$.

\section{Chlamydia as an example of a curable STI}

We calculated the three reproduction numbers using chlamydia as an example of a curable STI. In the baseline scenario, we assumed an infectious duration for asymptomatic chlamydia of one year (Molano et al., 2005; Heijne et al., 2011) (Table 2). The transmission probability was calibrated to a baseline prevalence of 3\% (as measured in Natsal-2 in 18 - 24 years old (Fenton et al., 2001)). For simplicity we assumed no period of immunity after natural clearance and equal natural clearance rates between men and women in the baseline scenario. Using these baseline values, we obtained the following reproduction numbers for chlamydia: $R_{0}=1.02 ; R_{c}=0.71$; and $R_{p}=1.15$.

We varied one parameter at a time to explore the sensitivity of the reproduction numbers to the infection and behavioural baseline values. For increasing prevalence (i.e. increasing transmission probability), the reproduction numbers increase (Fig. 3A). As demonstrated mathematically in Section 3.5, the three reproduction numbers have different threshold values above which chlamydia can persist in the population. Chlamydia can be endemically sustained even when the number of secondary cases is below 1 , because re-infection of the partner by the index case pushes $R_{0}$ above 1.

With increasing duration of infection (and constant transmission probability), all reproduction numbers increase because prevalence increases (Fig. 3B). For short infectious durations, $R_{p}$ can be smaller than $R_{0}$, because an individual has the potential to infect the same partner more than 
once, but the probability of still being infected when forming a new partnership is small. For long infection durations, the $R_{c}$ becomes higher than 1 because there is a high probability that a person separates from a partnership still being infected, so chlamydia can be transmitted to new partners.

The case reproduction number is not sensitive to the period of immunity (Fig. 3C). This is because $R_{c}$ only counts whether or not there is transmission to the susceptible partner, not the number of transmissions (i.e. $\epsilon$ is absent in Eq. 12). When there is no immunity, $R_{0}$ and $R_{p}$ are highest because, after natural clearance, a person can potentially become re-infected at the next sex act. For longer periods of immunity, the probability of re-infection of the index case within partnerships is low and $R_{p}$ approaches $R_{0}$. For very long durations of immunity (i.e. a SI type of infection), $R_{p}$ and $R_{0}$ approach $R_{c}$.

For very short partnership durations, the three reproduction numbers are the same (Fig. 3D). This is also shown mathematically in Section 3.4 when assuming instantaneous contacts. There is an optimum partnership duration in which the reproduction numbers are maximised. This duration is different for the three reproduction numbers. For longer partnership durations, the transmission probability is not high enough to sustain continuing transmission. Furthermore, the probability that the partner is re-infected becomes higher so the difference between $R_{0}$ and $R_{c}$ becomes bigger. Note that, when the partnership duration is changed and the rest of the parameters are kept at the same level, the number of new partners per year and the percentage of people in a partnership change. For short durations of partnerships, the number of new partners per year is high and few people are in a partnership and for high partnership duration, the number of new partners per year is low and many people are in a partnership.

When we changed the duration of infection, but kept the prevalence fixed at 3\% (by changing the transmission probability for every infectious duration), $R_{0}$ is (as expected) always above 1 and increases slowly with increasing duration of infection (Fig. 4). For short durations of infection, the probability of leaving the partnership and still being infected is low so the case reproduction number is low. The difference between $R_{c}$ and $R_{0}$ becomes smaller for longer duration of infection and $R_{c}$ will eventually approach $R_{0}$.

There is a combination of duration of infection and transmission probability that maximizes 
$R_{p}$ (Fig. 4). For the baseline behavioural parameters and prevalence, the $R_{p}$ is at a maximum for a duration of infection of 482 days and a transmission probability of 0.064 per sex act. Both estimates are within the ranges recently estimated for these parameters for chlamydia (Althaus et al., 2010, 2012). The duration of infection at which $R_{p}$ is maximised increases for increasing duration of immunity. The timing of the peak for $R_{p}$ is hardly influenced by the choice of the steady state prevalence (results not shown).

\section{Discussion}

We have introduced two new concepts, the case reproduction number $\left(R_{c}\right)$ and the partnership reproduction number $\left(R_{p}\right)$. We show that, for a curable STI, the average number of secondary cases an infected individual infects does not always equal the average number of secondary infections because of re-infection within partnerships. Values of $R_{c}$ and $R_{p}$ approach $R_{0}$ for long durations of immunity after natural clearance or if partnership durations are very short.

The main strength of this work is that we make a clear distinction between the average number of secondary infections $\left(R_{0}\right)$ and the average number of secondary cases (persons, $R_{c}$ ) an infected individual produces during his or her infectious period. This is important for a curable STI because $R_{c}$ allows the contribution of re-infection within partnerships to the transmission dynamics of the infection to be quantified. We showed that re-infection can push the basic reproduction number above 1 . The case reproduction number can have an epidemic threshold value that is different from 1 , which might be seen as a limitation. These values however, are important for the correct interpretation of reproduction numbers calculated from contact tracing data, which estimate the case reproduction number. For example, the reproduction number of 0.55 for chlamydia estimated by Potterat et al. (1999) was reported as a basic reproduction number, suggesting a declining epidemic.

Our study shows that, even when the case reproduction number is below 1, the basic reproduction number can be above 1. For a curable STI like chlamydia, a case reproduction number below 1 does not necessarily mean that the epidemic is decreasing.

The distinction between $R_{0}$ and $R_{c}$ is not always made clear in modelling studies (Chick et al., 2000; Williams et al., 2006; Fraser, 2007; Cooper et al., 2012). For example Chick et al. (2000) state that they calculate the average number of secondary cases but present the number of secondary 
infections. Moreover, estimates of the number of secondary infections for a curable STI that are based on the formula $\beta c D$ should be interpreted with caution. Such estimates ignore re-infection within partnerships because of the underlying assumptions that partnerships are instantaneous.

The values we obtained for the basic reproduction number are close to 1, and the case reproduction number was only above 1 for long infectious periods. The reproduction numbers are close to 1 because our model represents a general population with low infection prevalence and homogeneous sexual behaviour. This is a reasonable assumption for an infection like chlamydia, that is not solely concentrated in a core-group with higher partner change rates, but is also present in individuals with none or only one partner in the last year (Fenton et al., 2001). For other curable STIs, such as gonorrhea, it is shown that core-groups are important in sustaining endemic infection (Hethcote and Yorke, 1984). Therefore, we also provided derivations for the reproduction numbers for a model that included a core-group with higher partner change rates. Individuals in high sexual activity classes have more partners with shorter durations so the reproduction numbers should be higher, but also more alike because re-infection within partnerships is less likely to happen. As a consequence, the contribution of re-infection within partnerships to endemicity will be lower.

For many curable STIs, it is not known whether there is a period of immunity after natural clearance and, if it exists, whether there is full or partial protection against new infections. In our study, a period of immunity pushes $R_{0}$ closer to 1 even for very short durations of immunity. Others have shown interesting results from instantaneous contact models that included partial immunity after natural clearance (Gomes et al., 2004; Sharomi and Gumel, 2009). Sharomi and Gumel (2009) showed that when there is a higher probability of repeated infection during partial protection, the model exhibits backward bifurcation and an endemic equilibrium for $R_{0}<1$ can exist. Gomes et al. (2004) showed that with partial immunity there is a 're-infection threshold' above which vaccination fails because of repeated infection. This underlines the importance of understanding immunity after natural clearance for curable STIs.

Our results might be relevant to other infections that do not provoke lasting immunity and are predominantly spread within households, such as the carriage of pathogenic bacteria that can lead to serious disease. Examples include Streptococcus pneumoniae and Neisseria meningiditis. 
In households, people can infect the same household member several times. For these types of infections, the reproduction number is usually reported from the perspective of the household, i.e. the number of secondary households infected by one infected household (Pellis et al., 2009) rather than the individual. The partnership reproduction number resembles the household reproduction number because the perspective is shifted away from the individual level. However, $R_{p}$ also takes into account the additional number of secondary cases an index case produces because of clearance in a partnership and re-infection of the index case by the infected partner, which is not taken into account in the household reproduction number.

The differences between the basic and case reproduction numbers have implications for mathematical modelling studies and for field epidemiologists. We suggest that the precise meaning of reproduction numbers and how re-infection is incorporated should be stated in studies of curable STIs. Furthermore, field epidemiologists who aim to estimate reproduction numbers for a curable STI from contact tracing data should be aware that only the number of secondary cases $R_{c}$, not infections, can be calculated and that this reproduction number can have an epidemic threshold value that is different from 1. The reproduction numbers presented here can be used to quantify the fraction of the endemic prevalence of curable STIs that is attributable to re-infection. This quantification could lead to the identification of groups amongst whom re-infection is contributing substantially to endemic prevalence and where prevention of re-infection should be prioritised. Finally, this study shows that re-infection within partnerships means that curable STIs can be sustained endemically even when the average number of secondary cases a person produces during his or her infectious period is below one. 


\section{Acknowledgments}

This work was supported by the Swiss National Science Foundation grant numbers 320030135654 (JCMH), PDFMP3-124952 (SAH) and PZ00P3-136737 (CLA).

\section{References}

Althaus, C.L., Heijne, J.C., Roellin, A., Low, N., 2010. Transmission dynamics of Chlamydia trachomatis affect the impact of screening programmes. Epidemics 2, 123-131, doi:10.1016/j.epidem.2010.04.002.

Althaus, C.L., Heijne, J.C.M., Low, N., 2012. Towards more robust estimates of the transmissibility of chlamydia trachomatis. Sex Transm Dis 39, 402-404, doi:10.1097/OLQ.0b013e318248a550.

Anderson, R.M., May, R.M., 1982. Directly transmitted infections diseases: control by vaccination. Science 215, 1053-1060.

Anderson, R.M., May, R.M., 1991. Infectious Diseases of Humans: Dynamics and Control. Oxford University Press, Oxford.

Batteiger, B.E., Xu, F., Johnson, R.E., Rekart, M.L., 2010. Protective immunity to Chlamydia trachomatis genital infection: evidence from human studies. J Infect Dis 201 Suppl 2, S178-89.

Britton, T., Nordvik, M.K., Liljeros, F., 2007. Modelling sexually transmitted infections: the effect of partnership activity and number of partners on R0. Theor Popul Biol 72, 389-99, doi:10.1016/j.tpb.2007.06.006.

Brunham, R.C., Nagelkerke, N.J., Plummer, F.A., Moses, S., 1994. Estimating the basic reproductive rates of Neisseria gonorrhoeae and Chlamydia trachomatis: the implications of acquired immunity. Sex Transm Dis 21, 353-6.

Centers for Disease Control and Prevention, 2010. Sexually transmitted diseases treatment guidelines, 2010. MMWR Recomm Rep 59, 1-110. 
Centers for Disease Control and Prevention (CDC), 2010. Sexually Transmitted Disease Surveillance 2009. U.S. Department of Health and Human Services, Centers for Disease Control and Prevention, Atlanta, GA.

Chen, M.I., Ghani, A.C., 2010. Populations and partnerships: insights from metapopulation and pair models into the epidemiology of gonorrhoea and other sexually transmitted infections. Sex Transm Infect 86, 433-9, doi:10.1136/sti.2009.040238.

Chick, S.E., Adams, A.L., Koopman, J.S., 2000. Analysis and simulation of a stochastic, discreteindividual model of STD transmission with partnership concurrency. Math Biosci 166, 45-68.

Cooper, B.S., Kypraios, T., Batra, R., Wyncoll, D., Tosas, O., Edgeworth, J.D., 2012. Quantifying type-specific reproduction numbers for nosocomial pathogens: evidence for heightened transmission of an Asian sequence type 239 MRSA clone. PLoS Comput Biol 8, e1002454, doi:10.1371/journal.pcbi.1002454.

Diekmann, O., De Jong, M.C.M., Metz, J.A.J., 1998. A Deterministic Epidemic Model Taking Account of Repeated Contacts between the Same Individuals. J Appl Probab 35, 448-462.

Diekmann, O., Dietz, K., Heesterbeek, J.A., 1991. The basic reproduction ratio for sexually transmitted diseases: I. Theoretical considerations. Math Biosci 107, 325-339.

Diekmann, O., Heesterbeek, J.A.P., 2000. Mathematical Epidemiology of Infectious Diseases: Model Building, Analysis, and Interpretation. John Wiley, Chichester.

Dietz, K., 1975. Epidemiology. SIAM, Philadelphia. chapter Transmission and control of arbovirus diseases. pp. 104-121.

Dietz, K., Hadeler, K.P., 1988. Epidemiological models for sexually transmitted diseases. J Math Biol 26, 1-25.

Fenton, K.A., Korovessis, C., Johnson, A.M., McCadden, A., McManus, S., Wellings, K., Mercer, C.H., Carder, C., Copas, A.J., Nanchahal, K., Macdowall, W., Ridgway, G., Field, J., Erens, B., 2001. Sexual behaviour in Britain: reported sexually transmitted infections and prevalent genital 
Chlamydia trachomatis infection. Lancet 358, 1851-1854, doi:10.1016/S0140-6736(01)068866.

Ferguson, N.M., Keeling, M.J., Edmunds, W.J., Gani, R., Grenfell, B.T., Anderson, R.M., Leach, S., 2003. Planning for smallpox outbreaks. Nature 425, 681-685, doi:10.1038/nature02007.

Fraser, C., 2007. Estimating individual and household reproduction numbers in an emerging epidemic. PLoS One 2, e758, doi:10.1371/journal.pone.0000758.

Garnett, G.P., Anderson, R.M., 1996. Sexually transmitted diseases and sexual behavior: insights from mathematical models. J Infect Dis 174 Suppl 2, S150-61.

Gomes, M.G.M., White, L.J., Medley, G.F., 2004. Infection, reinfection, and vaccination under suboptimal immune protection: epidemiological perspectives. J Theor Biol 228, 539-549, doi:10.1016/j.jtbi.2004.02.015.

Heijne, J.C.M., Althaus, C.L., Herzog, S.A., Kretzschmar, M., Low, N., 2011. The role of reinfection and partner notification in the efficacy of Chlamydia screening programs. J Infect Dis 203, 372-7, doi:10.1093/infdis/jiq050.

Hethcote, H.W., Yorke, J.A., 1984. Gonorrhea transmission dynamics and control. Lecture Notes in Biomathematics, Springer-Verlag, Berlin.

Holmes, K.K., Sparling, P.F., Stamm, W.E., Piot, P., Wasserheit, J.N., Corey, L., Cohen, M.S., Watts, D.H., 2008. Sexually Transmitted Diseases. 4th edition ed., McGraw-Hill, New York.

Johnson, A.M., Mercer, C.H., Erens, B., Copas, A.J., McManus, S., Wellings, K., Fenton, K.A., Korovessis, C., Macdowall, W., Nanchahal, K., Purdon, S., Field, J., 2001. Sexual behaviour in Britain: partnerships, practices, and HIV risk behaviours. Lancet 358, 1835-1842, doi:10.1016/S0140-6736(01)06883-0.

Jolly, A.M., Wylie, J.L., 2002. Gonorrhoea and chlamydia core groups and sexual networks in Manitoba. Sex Transm Infect 78 Suppl 1, i145-51. 
Kretzschmar, M., Dietz, K., 1998. The effect of pair formation and variable infectivity on the spread of an infection without recovery. Math Biosci 148, 83-113.

Kretzschmar, M., Jager, J.C., Reinking, D.P., Van Zessen, G., Brouwers, H., 1994. The basic reproduction ratio $\mathrm{R} 0$ for a sexually transmitted disease in a pair formation model with two types of pairs. Math Biosci 124, 181-205.

Lloyd-Smith, J.O., Getz, W.M., Westerhoff, H.V., 2004. Frequency-dependent incidence in models of sexually transmitted diseases: portrayal of pair-based transmission and effects of illness on contact behaviour. Proc Biol Sci 271, 625-34, doi:10.1098/rspb.2003.2632.

Molano, M., Meijer, C.J.L.M., Weiderpass, E., Arslan, A., Posso, H., Franceschi, S., Ronderos, M., Munoz, N., van den Brule, A.J.C., 2005. The natural course of Chlamydia trachomatis infection in asymptomatic Colombian women: a 5-year follow-up study. J Infect Dis 191, 907-916, doi:10.1086/428287.

Pellis, L., Ferguson, N.M., Fraser, C., 2009. Threshold parameters for a model of epidemic spread among households and workplaces. J R Soc Interface 6, 979-987, doi:10.1098/rsif.2008.0493.

Potterat, J.J., Zimmerman-Rogers, H., Muth, S.Q., Rothenberg, R.B., Green, D.L., Taylor, J.E., Bonney, M.S., White, H.A., 1999. Chlamydia transmission: concurrency, reproduction number, and the epidemic trajectory. Am J Epidemiol 150, 1331-9.

Sharomi, O., Gumel, A.B., 2009. Re-infection-induced backward bifurcation in the transmission dynamics of Chlamydia trachomatis. Journal of Mathematical Analysis and Applications 356, 96-118.

Stigum, H., Falck, W., Magnus, P., 1994. The core group revisited: the effect of partner mixing and migration on the spread of gonorrhea, Chlamydia, and HIV. Math Biosci 120, 1-23.

Van de Velde, N., Brisson, M., Boily, M.C., 2010. Understanding differences in predictions of HPV vaccine effectiveness: A comparative model-based analysis. Vaccine 28, 5473-84, doi:10.1016/j.vaccine.2010.05.056. 
Williams, B.G., Lloyd-Smith, J.O., Gouws, E., Hankins, C., Getz, W.M., Hargrove, J., de Zoysa, I., Dye, C., Auvert, B., 2006. The potential impact of male circumcision on HIV in Sub-Saharan Africa. PLoS Med 3, e262, doi:10.1371/journal.pmed.0030262.

Xiridou, M., Geskus, R., De Wit, J., Coutinho, R., Kretzschmar, M., 2003. The contribution of steady and casual partnerships to the incidence of HIV infection among homosexual men in Amsterdam. AIDS 17, 1029-1038, doi:10.1097/01.aids.0000050879.72891.1d. 


\begin{tabular}{ll}
\hline Term & Definition \\
\hline$R_{c}$ & $\begin{array}{l}\text { Average number of secondary infected individuals (cases) one typical infected } \\
\text { individual will produce during his or her infectious period starting in a } \\
\text { partnership with two infected individuals in a totally susceptible population }\end{array}$ \\
& $\begin{array}{l}\text { Average number of secondary partnerships consisting of two infected } \\
\text { individuals one typical infected individual will produce during his or her } \\
\text { infectious lifetime starting in a partnership with two infected individuals } \\
\text { in a totally susceptible population }\end{array}$ \\
& $\begin{array}{l}\text { Average number of secondary infections one typical infected individual will } \\
\text { produce during his or her infectious period starting in a partnership with } \\
\text { two infected individuals in a totally susceptible population }\end{array}$ \\
$R_{0}$ & $\begin{array}{l}\text { Duration of the initial infectious period } \\
\text { Infectious period }\end{array}$ \\
Infectious lifetime & $\begin{array}{l}\text { Sum of the duration of the initial infectious period and subsequent infectious } \\
\text { periods when the index case clears the infection in a partnership and is } \\
\text { re-infected by the partner before separation }\end{array}$ \\
&
\end{tabular}


Table 2: Overview of the parameters of the pair model with homogenous sexual behaviour and chlamydia specific baseline values

\begin{tabular}{lclc}
\hline Parameters & Symbol & Explanation & Baseline value \\
\hline Behavioural & $\rho$ & pair formation rate (per year) & $5.0^{a}$ \\
& $\sigma$ & separation rate (per year) & $1.9^{a}$ \\
& $1 / \mu$ & duration of sexual active life (years) & 9 \\
Infection & $\beta$ & number of unprotected sex acts (per week) & 1 \\
& $1 / \gamma_{f}$ & infectious duration in women (years) & $0.1^{b}$ \\
& $1 / \gamma_{m}$ & infectious duration in men (years) & 1 \\
& $1 / \epsilon$ & duration of immunity (years) & 1 \\
\hline
\end{tabular}

${ }^{a}$ based on 1.5 new partners per year and $70 \%$ in a partnership at any time ${ }^{b}$ calibrated to a baseline prevalence of $3 \%$ 


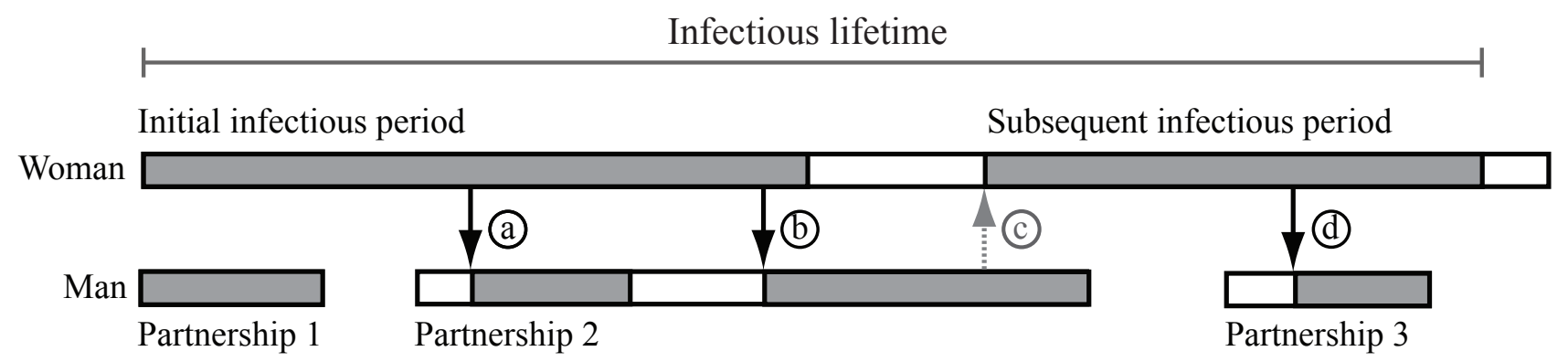

Figure 1: Illustration of how the female reproduction numbers are calculated for a SIS model. Grey bars denote infection and white bars denote the susceptible state. When an infected woman separates from her partnership and is still infected when forming a new partnership, she can transmit to her susceptible partner (a). Her partner can clear the infection and can become re-infected by the female index case (b). The same female index case can also clear the infection whilst in a partnership with an infected individual; she can then become re-infected by her partner (c). This re-infection prolongs the index case's infectious period. She is then able to infect a new partner because she is still infected when she forms a new partnership (d). A woman's infectious lifetime ends when she clears the infection while being single (as in this example) or when she clears the infection within the partnership and leaves the partnership being susceptible. In this example, the female case reproduction number $\left(R f_{c}\right)$ is 1 (only a), the basic reproduction number $\left(R f_{0}\right)$ is 2 $(\mathrm{a}+\mathrm{b})$, and the female partnership reproduction number $\left(R f_{p}\right)$ is also $2(\mathrm{a}+\mathrm{d})$. Please note that two infected partners cannot clear the infection at the same time. 


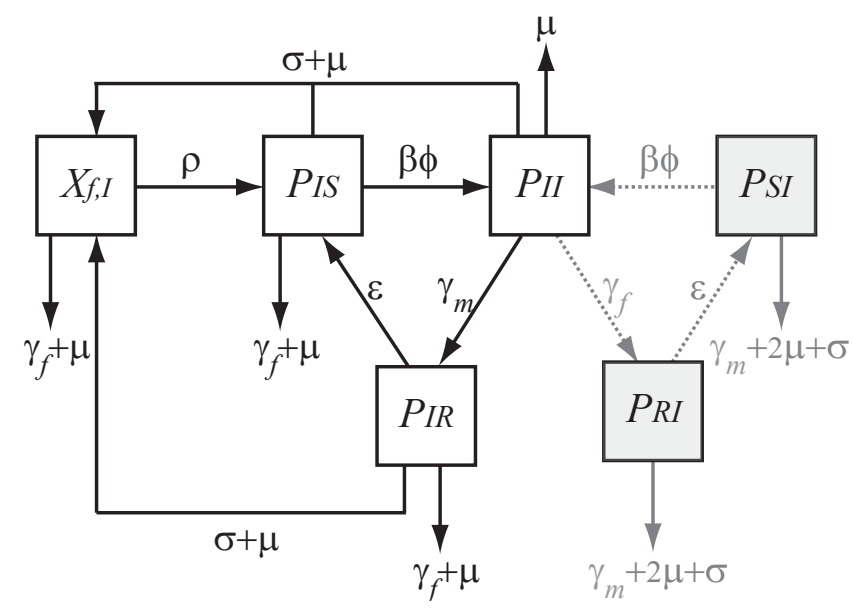

Figure 2: Flow diagram of the states of a SIRS pair model that includes an infectious woman (white boxes) or a susceptible woman with an infectious man (grey boxes). $X_{f, I}$ denotes single women who are infected and $P$ denotes pairs, where the first subscript denotes the infection status of the woman and the second the infection status of the man. Partnerships can be formed at rate $\rho$ and separate at rate $\sigma$. Transmission within partnerships occurs with a probability $\beta$ per sex act, assuming $\phi$ sex acts. The natural clearance rate of women is given by $\gamma_{f}$, and for men by $\gamma_{m}$. People lose their immunity at rate $\epsilon$ and become susceptible again. Please note that two infected partners cannot clear the infection at the same time. Individuals can leave the population at rate $\mu$. The dashed arrows denote the path where an infected woman clears the infection and becomes re-infected again in the same partnership. 
(a)

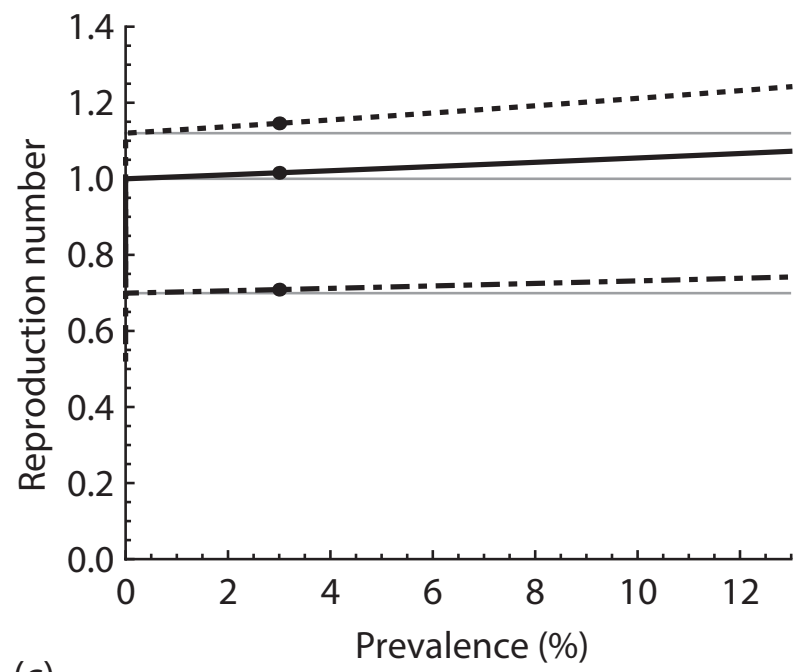

(c)

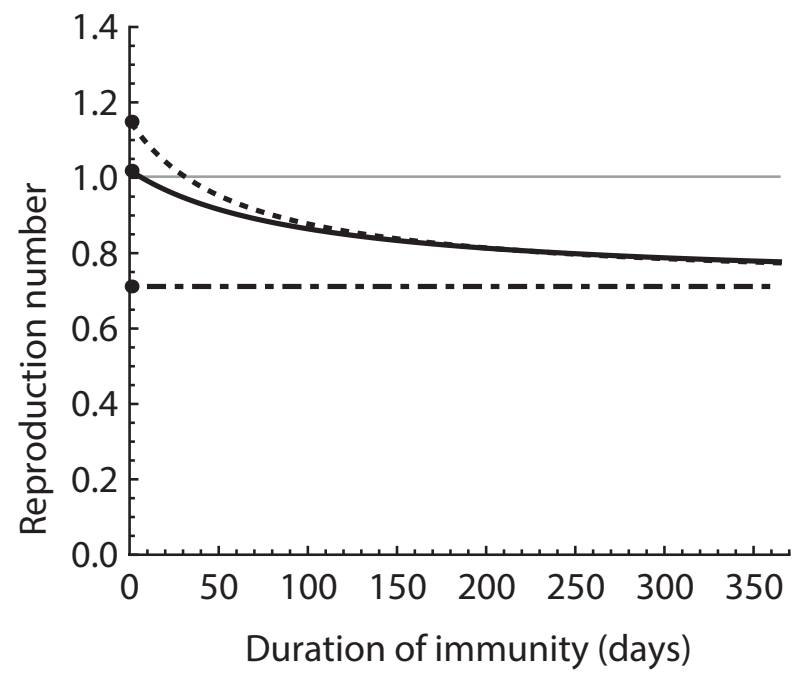

(b)

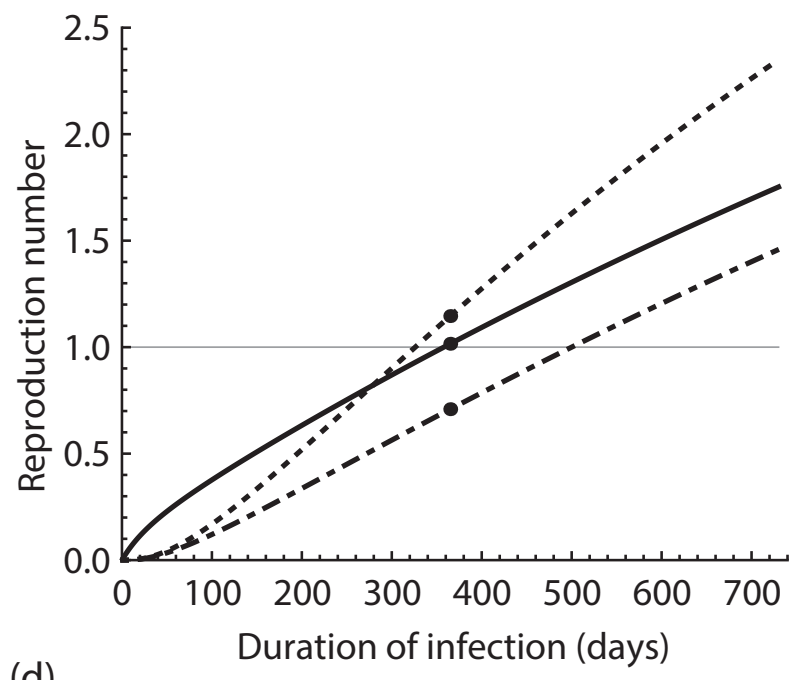

(d)

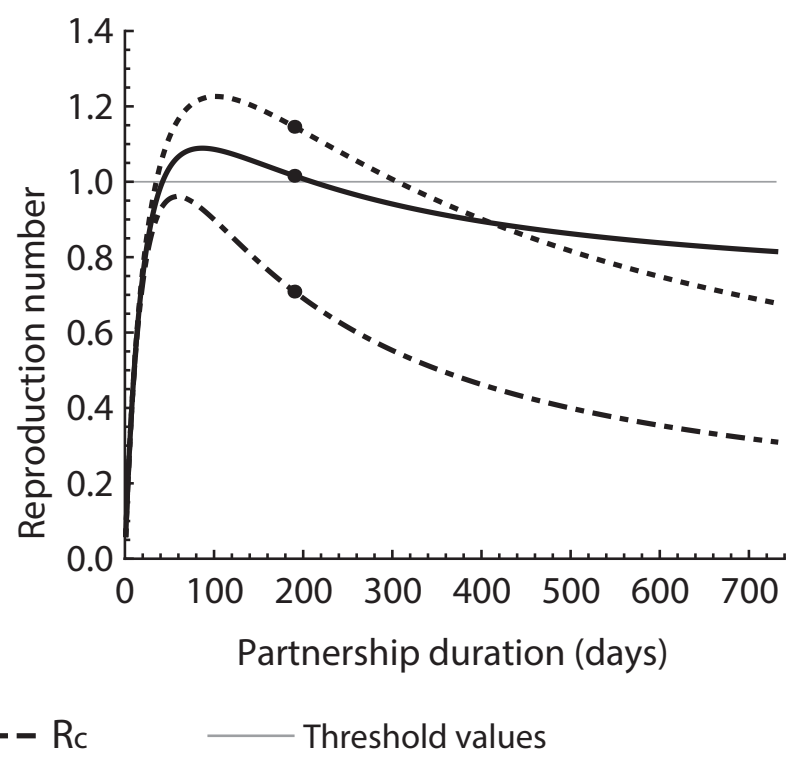

Figure 3: Effect of changing one parameter on the reproduction numbers and keeping all other parameters the same for an infection with characteristics similar to those of chlamydia. We changed the transmission probability (a), the duration of infection (assuming equal durations of infection between men and women) (b), the duration of immunity (c) and the duration of partnerships (d). Dashed lines indicate the partnership reproduction number $\left(R_{p}\right)$, black lines the basic reproduction number $\left(R_{0}\right)$ and dash-dotted lines the case reproduction numbers $\left(R_{c}\right)$. The grey lines indicate the epidemic threshold levels for all reproduction numbers (a) and for $R_{0}$ only (b-d). The dots indicate the baseline values from table 2 . 


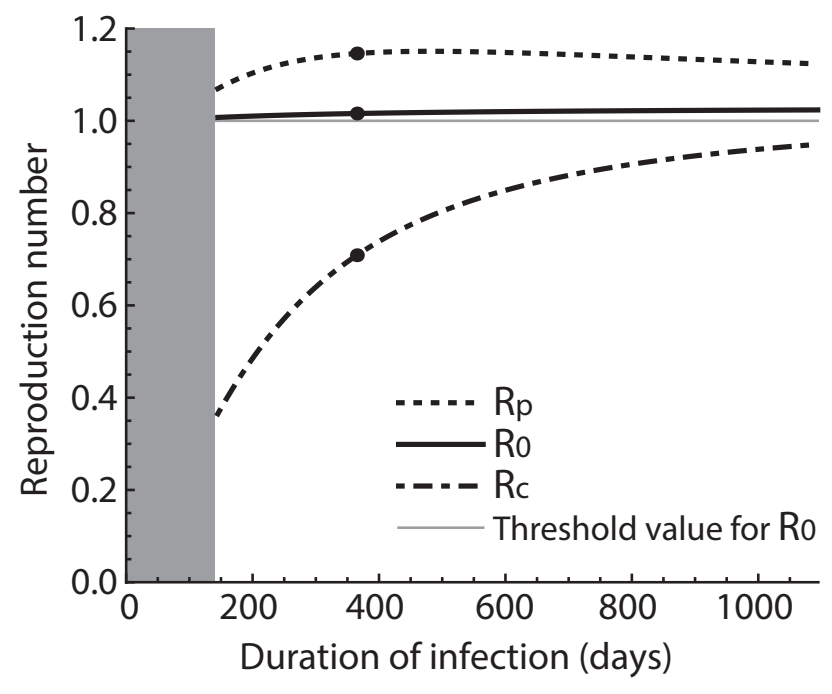

Figure 4: Effect of changing the duration of infection for a constant prevalence of $3 \%$ on the reproduction numbers for a SIS type of sexually transmitted infection. For every infectious duration, the transmission probability is changed to obtain the prevalence; all other parameters are kept constant. Infection durations shorter than 145 days could not be used because obtaining a 3\% prevalence resulted in a transmission probability per sex act that was bigger than one (grey area). Dashed lines indicate the partnership reproduction number $\left(R_{p}\right)$, black lines the basic reproduction number $\left(R_{0}\right)$ and dash-dotted lines the case reproduction numbers $\left(R_{c}\right)$. The grey line indicates the epidemic threshold level for $R_{0}$ and the dots the baseline values from table 2 . 


\section{AppendixA. Solutions for transmission probabilities}

We derived an expression for the transmission probability per sex act $\beta$ as a function of all parameters in the model and the prevalence $(\nu)$. The analytical result was derived in Mathematica (Wolfram Research, Inc., 2011, Champaign, Illinois. Version 8.0) using the Solve-function.

For a SIS type of infection, where the male and female natural clearance rate is equal (i.e. $\gamma_{f}=$ $\left.\gamma_{m}=\gamma\right)$, the transmission probability $\beta$ is

$$
\beta=-\frac{(\gamma+\mu)(2 \mu+\sigma)(2 \gamma+2 \mu+\sigma)(\gamma+2 \mu+\rho+\sigma)^{2}}{\left(\gamma^{2}(2 \mu+\sigma)^{2}+\gamma(2 \mu+\sigma)\left(6 \mu^{2}+2 \mu \rho+5 \mu \sigma+\sigma^{2}\right)+(2 \mu+\rho+\sigma)(x)\right) \phi}
$$

where

$$
x=4 \mu^{3}+(\nu-1) \rho \sigma^{2}+\mu \sigma(2(\nu-1) \rho+\sigma)+\mu^{2}(\nu \rho+4 \sigma)
$$

For a SIRS type of infection, the transmission probability $\beta$ as a function of the prevalence is obtained by setting the system of differential equations described in section 2 to zero. Because the analytic solution is lengthy, it is not shown here. However, the solution is available upon request from the author. 


\section{AppendixB. Heterogeneity in sexual behavior}

We extended the pair model to include two types of sexual activity classes (low and high) that differ in the preferred number of partners per year and the duration of partnerships. We assume that individuals are born as susceptible singles in an activity class and that they stay in the same class for the entire duration of their sexually active life. This differs from two other pair models that took heterogeneity in sexual behaviour into account (Kretzschmar et al., 1994; Xiridou et al., 2003). In Kretzschmar et al. (1994) all singles have the same pair formation rate, but upon formation of a partnership a certain fraction will be (short term) casual partnerships and all others are steady (long term) partnership. In Xiridou et al. (2003) all singles have the same pair formation rate for monogamous partnerships, but people can have an instantaneous contact while they are single and at a lower rate when they are in a partnership.

In the pair model with two sexual activity classes, the proportion of women and men in the high sexual activity class is assumed to be the same and is denoted by $p$. We defined two pair formation rates, one for individuals in the low activity class $\left(\rho_{L}\right)$ and one for individuals in the high activity class $\left(\rho_{H}\right)$. Mixing between the two classes occurs according to a mixing matrix $\mathbf{M}$ that can be set from fully assortative mixing to proportionate (random) mixing (Garnett and Anderson, 1996), taking into account the number of singles in each sexual activity class $\left(X_{L}\right.$ and $\left.X_{H}\right)$ and the pair formation rates

$$
\mathbf{M}=\left(m_{i j}\right)_{i, j \in\{L, H\}}=\left[\omega\left(\frac{\rho_{j} X_{j}}{\rho_{L} X_{L}+\rho_{H} X_{H}}\right)+(1-\omega) \delta_{i j}\right]_{i, j \in\{L, H\}}
$$

where $\delta_{i j}=1$ if $i=j$ and 0 otherwise and $\omega$ the proportion of contacts that will be proportionate or assortative ( $\omega=1$ for full random mixing, and $\omega=0$ for full assortative mixing). The separation rates depend on the sexual activity levels of both partners. The separation rate of two individuals in the low sexual activity classes is denoted by $\sigma_{L L}$ and in the high activity classes by $\sigma_{H H}$. We assume that $\sigma_{L H}=\sigma_{H L}$. The pair processes can be described by a set of differential equations, 
where the indices denote the sexual activity classes

$$
\begin{aligned}
\frac{d X_{L}}{d t} & =\mu(1-p) N+\left(\sigma_{L L}+\mu\right) 2 P_{L L}+\left(\sigma_{L H}+\mu\right) P_{L H}-\left(\rho_{L}+\mu\right) X_{L} \\
\frac{d X_{H}}{d t} & =\mu p N+\left(\sigma_{H H}+\mu\right) 2 P_{H H}+\left(\sigma_{L H}+\mu\right) P_{L H}-\left(\rho_{H}+\mu\right) X_{H} \\
\frac{d P_{L L}}{d t} & =\frac{1}{2} \rho_{L} m_{L L} X_{L}-\left(\sigma_{L L}+2 \mu\right) P_{L L} \\
\frac{d P_{L H}}{d t} & =\rho_{L}\left(1-m_{L L}\right) X_{L}-\left(\sigma_{L H}+2 \mu\right) P_{L H} \\
\frac{d P_{H H}}{d t} & =\frac{1}{2} \rho_{H} m_{H H} X_{H}-\left(\sigma_{H H}+2 \mu\right) P_{H H}
\end{aligned}
$$

Note that $\rho_{L}\left(1-m_{L L}\right) X_{L}=\rho_{H}\left(1-m_{H H}\right) X_{H}$. The mixing matrix $\mathbf{M}$ can be inferred by either running the system of differential equations into steady state, or analytically by assuming a closed population (i.e. $\mu=0$ ). The infection process is similar to that described in section 2 of the main text.

We present the derivation of the partnership reproduction number for a woman in the low activity class $\left(R f_{p}^{L}\right)$, using the same line of thinking as described in the main text. All other reproduction numbers are analogous. We start with an infected woman in a pair with an infected low risk male $\left(P_{I I}^{L L}\right)$ or in a pair with an infected high risk male $\left(P_{I I}^{L H}\right)$. The probability of her still being infected after separation from a partnership with another low risk partner $\left(q_{0 f}^{L L}\right)$ or from a partnership with a high risk partner $\left(q_{0 f}^{L H}\right)$ without re-infection is expressed as

$$
\begin{aligned}
q_{0 f}^{L L} & =\frac{\sigma_{L L}+\mu}{\sigma_{L L}+2 \mu+\gamma_{f}+\gamma_{m}}\left(1+\frac{\gamma_{m}}{\left(\sigma_{L L}+2 \mu+\gamma_{f}+\epsilon\right)}\left(1+\frac{\epsilon}{\sigma_{L L}+2 \mu+\gamma_{f}+\beta \phi}\right)\right) \mathrm{B} \\
q_{0 f}^{L H} & =\frac{\sigma_{L H}+\mu}{\sigma_{L H}+2 \mu+\gamma_{f}+\gamma_{m}}\left(1+\frac{\gamma_{m}}{\left(\sigma_{L H}+2 \mu+\gamma_{f}+\epsilon\right)}\left(1+\frac{\epsilon}{\sigma_{L H}+2 \mu+\gamma_{f}+\beta \phi}\right)\right)
\end{aligned}
$$

The probability that the male partner of a $P_{I I}^{L L}$ pair clears his infection and is then re-infected by his female partner is denoted by $r_{f}^{L L}$, and $r_{f}^{L H}$ denotes the probability of re-infection the male 


$$
\sum_{i=1}^{\infty} \sum_{k=0}^{i}\left(\begin{array}{l}
i \\
k
\end{array}\right)\left[q_{f}^{L L} m_{L L} d_{f}^{L}\right]^{k}\left[q_{f}^{L H}\left(1-m_{L L}\right) d_{f}^{L}\right]^{i-k}=\frac{q_{f}^{L L} m_{L L} d_{f}^{L}+q_{f}^{L H}\left(1-m_{L L}\right) d_{f}^{L}}{1-\left(q_{f}^{L L} m_{L L} d_{f}^{L}+q_{f}^{L H}\left(1-m_{L L}\right) d_{f}^{L}\right)}
$$

$$
\begin{aligned}
& s_{f}^{L L}=\left(\frac{\gamma_{f}}{\sigma_{L L}+2 \mu+\gamma_{f}+\gamma_{m}}\right)\left(\frac{\epsilon}{\sigma_{L L}+2 \mu+\gamma_{m}+\epsilon}\right)\left(\frac{\beta \phi}{\sigma_{L L}+2 \mu+\gamma_{m}+\beta \phi}\right) \\
& s_{f}^{L H}=\left(\frac{\gamma_{f}}{\sigma_{L H}+2 \mu+\gamma_{f}+\gamma_{m}}\right)\left(\frac{\epsilon \phi}{\sigma_{L H}+2 \mu+\gamma_{m}+\epsilon}\right)\left(\frac{\beta \phi}{\sigma_{L H}+2 \mu+\gamma_{m}+\beta \phi}\right)
\end{aligned}
$$

The probability of forming a partnership when the woman is still infectious and alive is different for a low risk single $\left(d_{f}^{L}\right)$ and a high risk single $\left(d_{f}^{H}\right)$

$$
d_{f}^{L}=\frac{\rho_{L}}{\rho_{L}+\gamma_{f}+\mu} \quad \text { and } \quad d_{f}^{H}=\frac{\rho_{H}}{\rho_{H}+\gamma_{f}+\mu}
$$

The probability that the index case forms a partnership with another low risk individual is $m_{L L}$, and with a high risk individual it is $m_{L H}=\left(1-m_{L L}\right)$. The total number of expected partners (both low risk and high risk) for a women in the low risk group during her infectious lifetime is now given as 
where $i$ is the total number of new partners and $k$ the number of partners that are with another low risk individual.

The probability that the woman transmits her infection to her susceptible partner depends on the duration of the partnership, so we define two different probabilities of transmission, depending on the type of partnership

$$
\begin{aligned}
b_{f}^{L} & =\frac{\beta \phi}{\sigma_{L L}+2 \mu+\gamma_{f}+\beta \phi} \\
b_{f}^{H} & =\frac{\beta \phi}{\sigma_{L H}+2 \mu+\gamma_{f}+\beta \phi}
\end{aligned}
$$

To obtain the expected number of secondary $P_{I I}^{L L}$ and $P_{I I}^{L H}$ partnerships during the infectious lifetime of a woman in the low risk group, we have to weight the transmission probabilities according to the expected number of partnership with a low risk male and with a high risk male

$$
R f_{p}^{L}=\left(b_{f}^{L} m_{L L}+b_{f}^{H}\left(1-m_{L L}\right)\right) \frac{q_{f}^{L L} m_{L L} d_{f}^{L}+q_{f}^{L H}\left(1-m_{L L}\right) d_{f}^{L}}{1-\left(q_{f}^{L L} m_{L L} d_{f}^{L}+q_{f}^{L H}\left(1-m_{L L}\right) d_{f}^{L}\right)}
$$

The derivation of the partnership reproduction number for low risk males towards the female population $R m_{p}^{L}$ and the partnership reproduction number for high risk females $\left(R f_{p}^{H}\right)$ and males $\left(R m_{p}^{H}\right)$ towards the opposite population is analogous. To obtain the case reproduction numbers, $s_{f}^{L L}$ and $s_{f}^{L H}$ should be set to zero. 